\title{
A non-doubling Trudinger inequality
}

\author{
by \\ Amiran Gogatishvili (Praha) and Pekka Koskela (Jyväskylä)
}

\begin{abstract}
We establish a Trudinger inequality for functions that satisfy a suitable Poincaré inequality in a Euclidean space equipped with a Borel measure that need not be doubling.
\end{abstract}

1. Introduction. It is by now well understood that a Poincaré inequality improves itself to a Sobolev-type inequality when we consider a doubling measure. To be more precise, suppose that a pair $u, g$ of measurable functions with $g \geq 0$ satisfies the inequality

$$
f_{B}\left|u-u_{B}\right| d \mu \leq C \operatorname{diam}(B)\left(f_{B} g^{p} d \mu\right)^{1 / p}
$$

for all balls $B$ in a metric space $X$. Here and in what follows, $f_{A}$ refers to $\mu(A)^{-1} \int_{A}, u_{A}$ is the average of $u$ over a set $A$, and we assume that $u$ is integrable on each ball $B$. Assume then that $\mu$ is doubling:

$$
\mu(B(x, 2 r)) \leq C_{d} \mu(B(x, r)),
$$

for each $x$ and all radii $r>0$. By iterating this inequality one obtains a lower estimate for the volume decay:

$$
\mu(B(x, r)) \geq C(r / R)^{s} \mu(B(x, R))
$$

whenever $B(x, r) \subset B(x, R)$. This exponent $s$ plays the role of the dimension: when $p<s$ and $g$ is $p$-integrable, the function $u$ is $q$-integrable for each $q<p s /(s-p)$, and when $p=s$ and $g$ is $s$-integrable, $u$ is exponentially integrable. Here $s$ can be replaced by any exponent as in (3), not necessarily obtained by iterating the doubling condition. These integrability results are in fact realized as inequalities. For example, for $q<p s /(p-s)$, one obtains

\section{Mathematics Subject Classification: Primary 46E35.}

The research for this paper was performed when A.G. was visiting the University of Jyväskylä financed by a researcher exchange grant program of the Academy of Sciences of the Czech Republic and the Academy of Finland and by the grant no. 201/01/0333 of the Grant Agency of the Czech Republic. He wishes to thank these organizations for the support. 
the inequality

$$
\left(f_{B}\left|u-u_{B}\right|^{q} d \mu\right)^{1 / q} \leq C^{\prime} \operatorname{diam}(B)\left(f_{2 B} g^{p} d \mu\right)^{1 / p},
$$

where $2 B$ is the ball with the same center as $B$ and of twice the radius of $B$. When the geometry of balls is sufficiently nice, the constant 2 can be omitted. Under the mild additional assumption that the space $X$ be connected, one even has a Trudinger inequality in the borderline case $p=s$. For these results see the papers [3], [4] by Hajłasz and Koskela.

A version of the improved regularity is also known to hold for certain nondoubling measures. The first result of this kind that we know of is from the paper [6] by Mateu, Mattila, Nicolau and Orobitg, where a John-Nirenberg type estimate is proven for functions in BMO. For motivations for relaxing the doubling assumption see [6] and the references therein.

Let us describe the non-doubling setting. Let $\mu$ be a non-negative Radon measure on $\mathbb{R}^{n}$, not necessarily doubling. We assume that $\mu(T)=0$ for each hyperplane $T$ orthogonal to one of the coordinate axes. In [6], the authors studied BMO for cubes with respect to such a measure and established an exponential integrability result. The point here is that cubes in $\mathbb{R}^{n}$ have special covering properties. An example was given in [6] to show that one cannot necessarily gain any improved integrability for functions in BMO if the measure fails to be doubling and the definition is given in terms of balls.

Subsequently, in [8], Orobitg and Pérez gave a version of the Sobolevtype inequalities for $p<s$. Here $s$ refers to a decay order of the measure, analogous to (3). It is natural to impose this assumption on the measure. As in the case of BMO, the Poincaré inequality was assumed to hold for cubes.

In this short note, we establish a version of the Trudinger inequality in the setting considered in [8].

THEOREM 1.1. Let $\mu$ be a non-negative Radon measure on $\mathbb{R}^{n}$ so that $\mu(T)=0$ for each hyperplane $T$ orthogonal to one of the coordinate axes. Assume that

$$
\mu(Q) \geq C_{\mu} \operatorname{diam}(Q)^{s}
$$

for each cube $Q$, where $s>1$. Suppose that $u$ is a locally integrable function so that

$$
f_{Q}\left|u-u_{Q}\right| d \mu \leq C \operatorname{diam}(Q)\left(f_{Q} g^{p} d \mu\right)^{1 / p}
$$

for each cube $Q$ with edges parallel to coordinate axes, where $g \in L_{\mu}^{s}\left(\mathbb{R}^{n}\right)$, and $1<p<s$. Then there exists a constant $C^{\prime}$ independent of $u, g$ such that 
for each cube as above we have

$$
\left\|u-u_{Q}\right\|_{\exp L^{s^{\prime}}(Q, \mu)} \leq C\left(\int_{Q} g^{s} d \mu\right)^{1 / s}
$$

where

$$
\|v\|_{\exp L^{s^{\prime}}(Q, \mu)}=\inf \left\{\lambda: f_{Q} \exp \left((g / \lambda)^{s^{\prime}}\right) d \mu<1\right\}
$$

and $s^{\prime}=s /(s-1)$.

The claim of Theorem 1.1 can be localized: if we assume (4) and (5) for each cube $Q \subset Q_{0}$, all with edges parallel to coordinate axes, then the claim holds for each analogous subcube of $Q_{0}$.

Our proof also gives the same integrability estimate for locally integrable functions $u$ that satisfy a.e. the pointwise estimate

$$
|u(x)-u(y)| \leq|x-y|(g(x)+g(y))
$$

with $g$ locally $s$-integrable. We believe that even this case is new. When $\mu$ is doubling, this class of functions introduced by Hajłasz in [2] coincides with the class of functions $u$ studied in Theorem 1.1. In our setting, the given pointwise estimate implies the indicated Poincaré inequality but the converse direction is doubtful.

Let us close this introduction by pointing out that we do not know if it is sufficient to assume the Poincaré inequality of Theorem 1.1 with exponent $s$. Our method, based on certain ideas from [6] and [8], breaks down if this inequality is taken as the starting point.

2. Proof of Theorem 1.1. We assume that $\mu$ is as in Theorem 1.1.

Fix a cube $Q_{0}$ and a point $x \in Q_{0}$. Let $0<r<\operatorname{diam}\left(Q_{0}\right)$. We define $\widetilde{Q}(x, r)$ as the unique cube with edges parallel to the coordinate axes and of diameter $r$, containing $x$, contained in $Q_{0}$, and with center as close to $x$ as possible. Clearly, for a fixed $x$, the function $\mu(\widetilde{Q}(x, r))$ is a continuous function of $r$ when $0<r<\operatorname{diam}\left(Q_{0}\right)$. Consequently, there exists a sequence $\left(r_{i}(x)\right)$ so that

$$
\mu\left(\widetilde{Q}\left(x, r_{i}(x)\right)\right)=2^{-i} \mu\left(Q_{0}\right) .
$$

Let us define $Q_{i}(x)=\widetilde{Q}\left(x, r_{i}(x)\right)$. Because $\widetilde{Q}(x, r) \subset \widetilde{Q}(x, R)$ when $r<R$, we have

$$
Q_{i+1}(x) \subset Q_{i}(x)
$$

for all $i$. Associate a sequence $\left(Q_{i}(x)\right)$ to each $x \in Q_{0}$ by the above procedure. 
We now define a maximal operator associated to our family of cubes by setting

$$
\widetilde{M}_{p} g(x)=\sup _{i \geq 0}\left(\frac{1}{\mu\left(Q_{i}(x)\right)} \int_{Q_{i}(x)}|g(y)|^{p} d \mu\right)^{1 / p} .
$$

Lemma 2.1. We have

$$
\mu\left(\left\{x \in Q_{0}: \widetilde{M}_{p} g(x)>\lambda\right\}\right) \leq b(n) \lambda^{-p} \int_{Q_{0}} g^{p} d \mu,
$$

where $b(n)$ only depends on $n$. Moreover, for each $0<q<p$,

$$
\left(\frac{1}{\mu\left(Q_{0}\right)} \int_{Q_{0}}\left(\widetilde{M}_{p} g\right)^{q} d \mu\right)^{1 / q} \leq 2^{1 / q}\left(\frac{q b(n)}{p-q}\right)^{1 / p}\left(\frac{1}{\mu\left(Q_{0}\right)} \int_{Q_{0}} g^{p} d \mu\right)^{1 / p}
$$

Estimates like the one in Lemma 2.1 are well known when either the measure is doubling or when the cubes in the definition of $\widetilde{M}_{p} g$ are centered at the point $x$. In the first case, the doubling allows one to effectively use the Vitali covering theorem and in the second case one relies on the Besicovitch covering theorem. Thus the second case is heavily Euclidean whereas the doubling assumption on the measure suffices in general metric measure spaces. In our setting, we cannot directly apply the Besicovitch covering theorem. The key idea in our argument comes from [6]. For the sake of completeness we give a rather detailed proof.

Proof of Lemma 2.1. For each $x \in E_{\lambda}:=\left\{x \in Q_{0}: \widetilde{M}_{p} g(x)>\lambda\right\}$ there is a cube $Q_{i(x)}(x)$ so that

$$
\left(\frac{1}{\mu\left(Q_{i(x)}(x)\right)} \int_{Q_{i(x)}(x)}|g(y)|^{p} d \mu\right)^{1 / p}>\lambda .
$$

We define $R_{i(x)}(x)$ as the unique rectangle in $\mathbb{R}^{n}$ centered at $x$ so that $R_{i(x)}(x) \cap Q_{0}=Q_{i(x)}(x)$. Denote by $\mathcal{R}$ the collection of rectangles obtained by this procedure. It follows from the definition that the ratio of any two side lengths of a rectangle in $\mathcal{R}$ is at most 2 . So, by the Besicovitch covering theorem (see [1]), we obtain a countable subcollection of rectangles $R_{i} \in \mathcal{R}$ covering $E_{\lambda}$ so that each point in $\mathbb{R}^{n}$ belongs to at most $b(n)$ of these rectangles, where $b(n)$ only depends on $n$. Therefore,

$$
\begin{aligned}
\mu\left(E_{\lambda}\right) & \leq \sum_{i=1}^{\infty} \mu\left(R_{j} \cap Q_{0}\right) \leq \lambda^{-p} \sum_{i=1}^{\infty} \mu\left(Q_{i}\right) \frac{1}{\mu\left(Q_{i}\right)} \int_{Q_{i}}|g|^{p} d \mu \\
& \leq \lambda^{-p} \sum_{i=1}^{\infty} \int_{Q_{i}}|g|^{p} d \mu \leq b(n) \lambda^{-p} \int_{E_{\lambda}}|g|^{p} d \mu .
\end{aligned}
$$


The second statement follows from the Kolmogorov inequality which states that every weak-type estimate as in the first part of the claim yields a strongtype inequality when the exponent $p$ is relaxed to $0<q<p$; this can be easily checked by hand by using the Cavalieri formula for Lebesgue integrals (cf. Theorem 14.11 in [4]).

We now give the proof of Theorem 1.1.

Proof of Theorem 1.1. Fix a point $x \in Q_{0}$ and the associated sequence $\left(Q_{i}(x)\right)$ of cubes. To simplify our notation we will mostly suppress the dependence of $Q_{i}$ on $x$ in what follows.

By the Lebesgue differentiation theorem (cf. [7]) we may assume that

$$
\lim _{i \rightarrow \infty} u_{Q_{i}(x)}=u(x)
$$

$\mu$-a.e. and hence we may assume that our fixed point $x$ has this property. Let $N=N(x)$ be a positive integer whose value will be determined later. Now

$$
\begin{aligned}
\frac{1}{2}\left|u(x)-u_{Q_{0}}\right| \leq & \frac{1}{2} \sum_{i=0}^{\infty}\left|u_{Q_{i}}-u_{Q_{i+1}}\right| \leq \sum_{i=0}^{\infty} \frac{1}{\mu\left(Q_{i}\right)} \int_{Q_{i}}\left|u-u_{Q_{i}}\right| d \mu \\
= & \sum_{i=0}^{N} \frac{1}{\mu\left(Q_{i}\right)} \int_{Q_{i}}\left|u-u_{Q_{i}}\right| d \mu \\
& +\sum_{i=N+1}^{\infty} \frac{1}{\mu\left(Q_{i}\right)} \int_{Q_{i}}\left|u-u_{Q_{i}(x)}\right| d \mu=: I_{1}+I_{2} .
\end{aligned}
$$

Furthermore, by (5), (4), (8), changing the order of summation, using (7) and the assumption $p<s$, and finally employing the Hölder inequality we see that

$$
\begin{aligned}
& \frac{C_{\mu}^{1 / s}}{C} I_{1} \leq C_{\mu}^{1 / s} \sum_{i=0}^{N} \operatorname{diam}\left(Q_{i}\right)\left(\frac{1}{\mu\left(Q_{i}\right)} \int_{Q_{i}} g^{p} d \mu\right)^{1 / p} \\
& \leq \sum_{i=0}^{N} \mu\left(Q_{i}\right)^{1 / s}\left(\frac{1}{\mu\left(Q_{i}\right)} \int_{Q_{i}} g^{p} d \mu\right)^{1 / p}=\sum_{i=0}^{N} \mu\left(Q_{i}\right)^{1 / s-1 / p}\left(\int_{Q_{i}} g^{p} d \mu\right)^{1 / p} \\
& =\sum_{i=0}^{N} \mu\left(Q_{i}\right)^{1 / s-1 / p}\left(\sum_{k=i}^{N} \int_{Q_{k} \backslash Q_{k+1}} g^{p} d \mu+\int_{Q_{N+1}} g^{p} d \mu\right)^{1 / p} \\
& \leq \sum_{i=0}^{N} \mu\left(Q_{i}\right)^{1 / s-1 / p} \sum_{k=i}^{N}\left(\int_{Q_{k} \backslash Q_{k+1}} g^{p} d \mu\right)^{1 / p} \\
& \quad+\sum_{i=0}^{N} \mu\left(Q_{i}\right)^{1 / s-1 / p}\left(\int_{Q_{N+1}} g^{p} d \mu\right)^{1 / p}
\end{aligned}
$$




$$
\begin{aligned}
\leq & \sum_{i=0}^{N}\left(\int_{Q_{i} \backslash Q_{i+1}} g^{p} d \mu\right)^{1 / p} \sum_{k=0}^{i} \mu\left(Q_{k}\right)^{1 / s-1 / p} \\
& +C^{\prime} \mu\left(Q_{N+1}\right)^{1 / s-1 / p}\left(\int_{Q_{N+1}} g^{p} d \mu\right)^{1 / p} \\
\leq & C^{\prime}\left(\sum_{i=0}^{N}\left(\int_{Q_{i} \backslash Q_{i+1}} g^{p} d \mu\right)^{1 / p} \mu\left(Q_{i}\right)^{1 / s-1 / p}\right. \\
& \left.+\mu\left(Q_{N+1}\right)^{1 / s-1 / p}\left(\int_{Q_{N+1}} g^{p} d \mu\right)^{1 / p}\right) \\
\leq & C^{\prime}\left(\sum_{i=0}^{N}\left(\int_{Q_{i} \backslash Q_{i+1}} g^{s} d \mu\right)^{1 / s}\right. \\
& \left.+\left(\int_{Q_{N+1}} g^{1 / s}\right)^{1 / C^{\prime}}\right)^{\prime} N(x)^{1 / s^{\prime}}\left(\int_{Q_{0}} g^{s} d \mu\right)^{1 / s},
\end{aligned}
$$

where $C^{\prime}=C^{\prime}(p, s)$. To estimate $I_{2}$ we first apply the lower decay order (4) of $\mu$ and the equality (7) to see that

$$
\operatorname{diam}\left(Q_{i}\right) \leq C_{\mu}^{-1 / s} \mu\left(Q_{i}\right)^{1 / s} \leq C 2^{-i / s} \mu\left(Q_{0}\right)^{1 / s}
$$

Taking this and the Poincaré inequality (5) into account results in the estimate

$$
\begin{aligned}
I_{2} & =\sum_{i=N+1}^{\infty} \frac{1}{\mu\left(Q_{i}\right)} \int_{Q_{i}}\left|u-u_{Q_{i}}\right| d \mu \\
& \leq C C_{\mu}^{-1 / s} \sum_{i=N(x)}^{\infty} 2^{-i / s} \mu\left(Q_{0}\right)^{1 / s}\left(\frac{1}{\mu\left(Q_{i}(x)\right)} \int_{Q_{i}} g^{p} d \mu\right)^{1 / p} \\
& \leq C C_{\mu}^{-1 / s} \sum_{i=N+1}^{\infty} 2^{-i / s} \mu\left(Q_{0}\right)^{1 / s}\left(\frac{1}{\mu\left(Q_{i}\right)} \int_{Q_{i}} g^{s} d \mu\right)^{1 / s} \\
& \leq C^{\prime}\left(C, s, C_{\mu}\right) 2^{-N(x) / s} \mu\left(Q_{0}\right)^{1 / s} \widetilde{M}_{s} g(x) .
\end{aligned}
$$

We now choose $N(x)$ to be the integer part of

$$
s \log _{2}\left(\frac{\widetilde{M}_{s} g(x)}{\left(f_{Q_{0}} g^{s} d \mu\right)^{1 / s}}\right) .
$$


With this choice inequalities (11)-(13) give us the estimate

$$
\begin{aligned}
& \left|u(x)-u_{Q_{0}}\right| \\
& \leq C^{\prime \prime}\left(\log _{2}\left(\frac{\left(\widetilde{M}_{s} g(x)\right)^{s}}{\frac{1}{\mu\left(Q_{0}\right)} \int_{Q_{0}} g^{s} d \mu}\right)\right)^{1 / s^{\prime}}\left(\int_{Q_{0}} g^{s} d \mu\right)^{1 / s}+C^{\prime \prime}\left(\int_{Q_{0}} g^{s} d \mu\right)^{1 / s},
\end{aligned}
$$

where $C^{\prime \prime}=C\left(p, s, C_{\mu}, C\right)$. Consequently, for $\lambda>0$,

$$
\int_{Q_{0}} \exp \left(\log 2\left(\frac{\left|u(x)-u_{Q_{0}}\right|}{\lambda C^{\prime \prime}\left(\int_{Q_{0}} g^{s} d \mu\right)^{1 / s}}\right)^{s^{\prime}}\right) d \mu \leq 2^{1 / \lambda} \int_{Q_{0}} \frac{\left(\widetilde{M}_{s} g(x)\right)^{s / \lambda} d \mu}{\left(\frac{1}{\mu\left(Q_{0}\right)} \int_{Q_{0}} g^{s} d \mu\right)^{1 / \lambda}} .
$$

The claim of Theorem 1.1 follows by employing the boundedness of the maximal operator given by Lemma 2.1, which can be applied when $\lambda>1$.

REMARK 2.2. The proof of Theorem 1.1 above shows that we actually proved the Trudinger-type estimate for the potential

$$
J g(x)=\sum_{i=0}^{\infty} \operatorname{diam}\left(Q_{i}(x)\right)\left(\frac{1}{\mu\left(Q_{i}(x)\right)} \int_{Q_{i}(x)} g^{p} d \mu\right)^{1 / p} .
$$

\section{References}

[1] M. de Guzmán, Differentiation of Integrals in $\mathbb{R}^{n}$, Lecture Notes in Math. 481, Springer, Berlin, 1975.

[2] P. Hajłasz, Sobolev spaces on an arbitrary metric space, Potential Anal. 5 (1996), 403-415.

[3] P. Hajłasz and P. Koskela, Sobolev meets Poincaré, C. R. Acad. Sci. Paris 320 (1995), 1211-1215.

[4] -, -, Sobolev met Poincaré, Mem. Amer. Math. Soc. 145 (2000), no. 688.

[5] P. MacManus and C. Pérez, Trudinger inequalities without derivatives, Trans. Amer. Math. Soc. 354 (2002), 1997-2012.

[6] J. Mateu, P. Mattila, A. Nicolau and J. Orobitg, BMO for nondoubling measures, Duke Math. J. 102 (2000), 533-565.

[7] P. Mattila, Geometry of Sets and Measures in Euclidean Spaces: Fractals and Rectifiability, Cambridge Stud. Adv. Math. 44, Cambridge Univ. Press, Cambridge, 1995.

[8] J. Orobitg and C. Pérez, $A_{p}$ weights for nondoubling measures in $R^{n}$ and applications, Trans. Amer. Math. Soc. 354 (2002), 2013-2033.

Mathematical Institute

Academy of Sciences of the Czech Republic Žitna 25

11567 Praha 1, Czech Republic

E-mail: gogatish@math.cas.cz
Department of Mathematics and Statistics University of Jyväskylä P.O. Box 35 FIN-40014 Univ. of Jyväskylä, Finland E-mail: pkoskela@maths.jyu.fi 